\title{
FLOW-STRUCTURE INSTABILITY PREVENTION IN A MEMS HIGH FLOW GAS VALVE
}

\author{
Aaron J. Knobloch ${ }^{1}$, Charles E. Seeley ${ }^{2}$, Amol Mulay ${ }^{3}$, Richard J. Saia ${ }^{1}$ \\ ${ }^{1}$ Micro and Nano Structures Technologies \\ ${ }^{2}$ Energy and Propulsion Technologies \\ General Electric Global Research Center \\ Niskayuna, NY USA \\ ${ }^{3}$ John F. Welch Technology Centre \\ Bangalore, India
}

\begin{abstract}
This paper presents an innovative method of preventing structural resonance due to a fluid-structure interaction for a MEMS high flow rate gas valve. This normally closed gate valve design consists of a pair of slender electrothermal beam actuators that move a slider with rectangular slits to modulate the flow of gas through a series of flow channels below the actuator. Under certain flow and voltage conditions, the slider structure enters a resonance condition first identified by an audible noise and an undesirable flow output. Visual inspection of the slider and electrothermal beams during resonance indicated that the in-plane displacements of the normally quasi-static beams oscillate wildly such that the beams buckle. A computational fluid dynamic (CFD) analysis in combination with a mechanical model of the electrothermal beams showed that forces on the walls of the slots tend to close the actuator but are insufficient on their own to cause beam buckling. The design of the valve, with its "floating" slider mechanism, has no sliding contact points to create friction that could also create damping. Therefore, this structure has a high $\mathrm{Q}$ factor which can amplify the resonant oscillating displacement. Based on viscoelastic properties of RTV silicone, a small, precisely controlled amount of silicone was dispensed using a computer controlled dispense system to create two damping "straps" between the slider mechanism and the rigid supporting structure around the valve. Subsequent testing indicated that the instability could not be induced under normal operating conditions once the silicone dampers were installed.
\end{abstract}

\section{INTRODUCTION}

Many MEMS devices are based on oscillating microstructures such as comb drives, quartz crystal resonators and cantilever beams. These devices with size scales from nanometers up to hundreds of microns are able to achieve high actuation strokes due to a minimal amount of damping inherent in their design [1-2]. When used as sensors, an important factor to improving the sensitivity of these devices is to maximize the sharpness of resonance, or Q-factor. The Q-factor is heavily influenced by the ambient environment [3-4] since typically Q scales with viscosity of the fluid surrounding the MEMS device. In some cases, this necessitates vacuum operation for minimal viscous damping of the structure. While numerous studies of the effects of damping of MEMS oscillatory sensors and actuators have been presented, the majority of these have focused on increasing $Q$ for better performance. Few studies have examined reducing $\mathrm{Q}$ to increase damping on MEMS devices [5].

Extensive prior research on the design and microfabrication of MEMS devices for flow control of gases and liquids is available. One design that is ameable to high flow rate gas modulation is a gate valve structure [6]. Since a gate valve design actuates perpendicular to the direction of flow, a gate valve is easily integrated with planar electrothermal or electrostatic actuation schemes and does not require a high force actuator to work against the flow to close the valve. This paper examines a high flow rate gate microvalve that exhibits a flow-induced resonance from a coupled interaction of the fluid and structure (FSI) that adversely affects the valve performance. A novel method for adding damping to the microvalve is presented that mitigates the undesirable resonance from the fluid structure interaction.

\section{VALVE DESIGN AND OPERATION}

The objective of the microvalve design in this effort was to develop the capability of modulating airflow rates of 5000$10000 \mathrm{sccm}$. In order to minimize valve power consumption and maximize flow area, a gate valve design was utilized that operates by moving a set of fingers across a series of slots as shown in Figure 1. The required flow rates and die size constraints necessitate slot sizes on the order of $120 \mu \mathrm{m}$ in width [7]. Therefore, an actuation system was required to produce at least this amount of stroke within the geometric constraints of the die. A bent beam electrothermal actuator (Vbeam actuator) was chosen based on a survey of competing actuation concepts and prior experience with this design [8].

Figure 2 shows a top view of the microvalve structure. While the focus of this paper is on the flow induced instability and prevention rather than the fabrication, the following is a brief description of the microvalve design and process flow. The slider is composed of 30 slots $120 \mu \mathrm{m}$ wide and $2990 \mu \mathrm{m}$ long. The electrothermal actuator is a bent beam $35 \mu \mathrm{m}$ wide, $6900 \mu \mathrm{m}$ long. The actuator is fabricated on a $385 \mu \mathrm{m}$ thick highly doped $(0.015-$ $0.02 \mathrm{ohm}-\mathrm{cm})$ silicon wafer with a two sided deep reactive ion

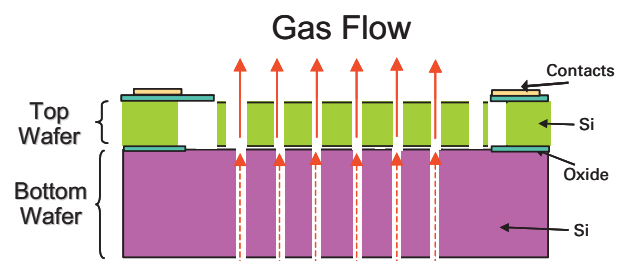

Figure 1. Cross-sectional view of the MEMS gas valve where the middle slider composed of an array of $135 \mu \mathrm{m}$ wide fingers uncovering the slots of bottom wafer.

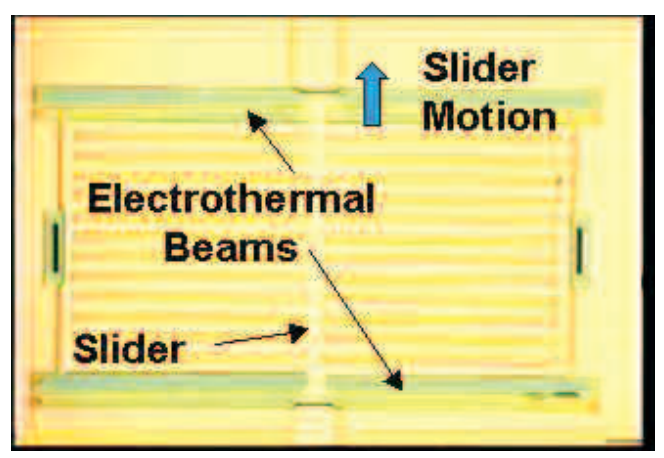

Figure 2. Top view of the early generation MEMS gas valve showing an array of slots actuated by a pair of electrothermal actuators. 


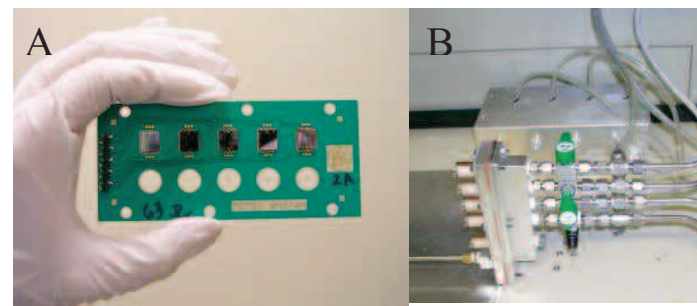

Figure 3. A, picture of the microvalve assembled on an aluminum board. B, microvalve test manifold with individual solenoids controlling each channel (orifice not shown).

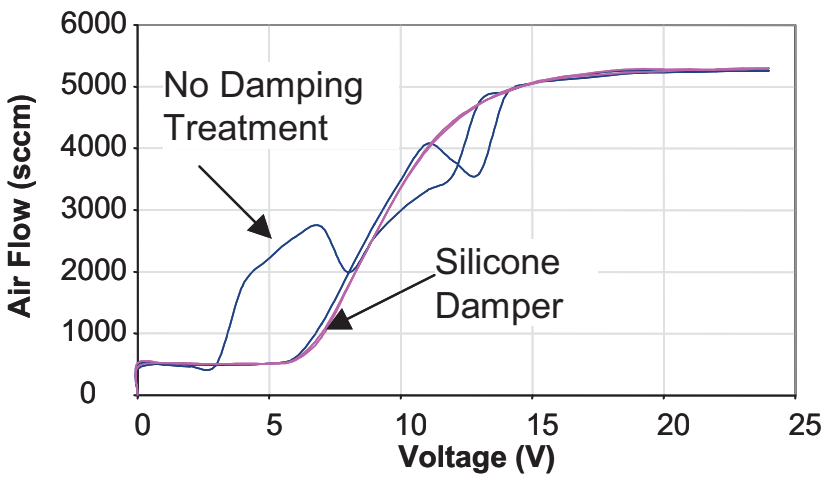

Figure 4. Comparison between undamped and damped gas valve operation.

etch. The actuator wafer is silicon fusion bonded to another wafer etched with $30120 \mu \mathrm{m}$ wide slots such that the slots are covered in the non-energized position. A short etch in the actuator and slot regions prevents a fusion bond between the slider or actuator and the lower wafer.

Since the microvalve is thermally actuated, a Thermal $\mathrm{Clad}^{\circledR}$ circuit board with an aluminum substrate and dielectric clad top from The Bergquist Company was chosen as a test vehicle due to its heat dissipation properties (See Figure 3). The die are mounted over open slots in the board using standard die pick and place equipment and are then wire bonded to make electrical connections. Each die is adhered to the board using GE RTV116 high temperature silicone. This compliant material with good thermal conductivity maintains its mechanical properties at elevated temperature (die temperatures can reach upwards of $100^{\circ} \mathrm{C}$ ). A populated board is inserted into a two part metal manifold with machined flow passages and solenoid shutoff valves for each microvalve. The manifold is part of a fluidic test rig (Figure 3) with integrated flow meters and an upstream pressure regulator $(1245 \mathrm{~Pa})$ for microvalve characterization. Pressure is measured in the flow circuit just upstream to the set of microvalves at the manifold inlet and just downstream of each microvalve with a differential pressure sensor to determine the pressure drop across each valve. Following the downstream pressure sensor is a restrictive $4.9 \mathrm{~mm}$ diameter orifice.

In order to characterize the flow response of the microvalve, the input voltage to the valve was ramped up from $0 \mathrm{~V}$ to $24 \mathrm{~V} \mathrm{DC}$ and back down to $0 \mathrm{~V}$ in increments of $1 \mathrm{~V}$. Once the voltage was set, a time delay of four seconds was imposed for the flow to stabilize before pressure and flow measurements were taken. A software-controlled procedure was used to ramp the voltage, open/close the solenoid shutoff valves and record the data as needed. Figure 4 shows the flow response of a typical microvalve without damping treatment along side the desired response. The instability seen in Figure 4 is not consistent valve to valve and a very small percentage $(<10 \%)$ of valves exhibited no audible noise

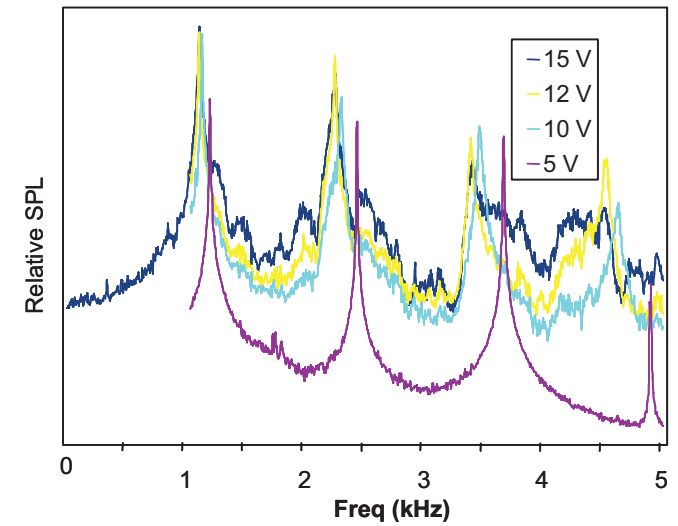

Figure 5. Spectrum analysis of the gas valve noise at a variety of voltages.

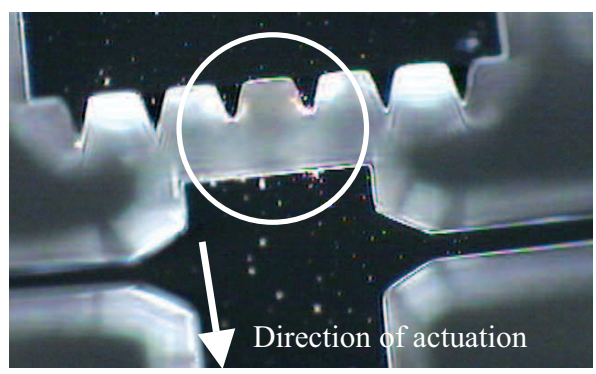

Figure 6. Close up view of valve and reverse stop after operation of unstable valve. The silicon chips and divots on the actuator along with the blunted points indicate actuation of the valve opposite to the intended direction.

or flow disturbance. The flow instability was especially noticeable in the range of voltage from $\sim 5 \mathrm{~V}$ to $14 \mathrm{~V}$. Audibly, the noise was characterized by single frequency flute like tone with an extra "harshness" and a mechanical sounding vibration. A typical spectrum of the tone is shown in Figure 5. The tone was recorded by placing a microphone near the valve and calculating the autospectrum of the frequency content of the acoustic signal. Although the amplitude is strictly relative in this figure, the total RMS level of the sound is estimated at $80-90 \mathrm{~dB}$ SPL. The acoustic spectrum indicates a fundamental frequency at approximately $1.2 \mathrm{kHz}$, and a number of $\mathrm{N}$ multiple harmonics. This is consistent with the expected fluid structure interaction. A resonance condition is created where the motion of the fluid excites the slider structure in a periodic manner causing the valve to open and close at a rapid pace. A pressure wave is created with each opening and closing as the fluid varies in velocity. This pressure wave creates the tone observed by the listener. The pressure wave is also "chopped" by the motion of the slider, so it is not a pure sine wave and creates the $\mathrm{N}$ multiple harmonics that give the harsh tone. The mechanical vibration noise is most likely the slider contacting the sides of the MEMS device.

Inspection of the valves after operation (Figure 6) revealed noticeable damage that would require bending of the beams in the opposite direction as is intended. Video of the valve's operation was taken with the backend of the manifold removed from the assembly. This video confirmed a resonance in the structure at valve positions corresponding to the audible noise. The oscillation amplitude is observed to vary from the maximum beam displacement at a particular input voltage to a negative displacement causing contact with the reverse stop. This reverse displacement causes the two beams to have an S-shape implying beam buckling. This resonance behavior was observed on more 
than 30 devices from a multitude wafers and wafer lots eliminating process variability as a root cause.

\section{FLUIDIC AND STRUCTURAL MODELING}

Analysis of the conditions causing the electrothermal beams to lose stiffness and become susceptible to the destructive FSI is composed of two parts: a fluidic model of the forces acting on the actuator and a mechanical model of the forces required to buckle the beam. A computational fluid dynamics (CFD) model was constructed using the software package FLUENT to determine the forces created by the flow conditions through the valve. The initial single slot two-dimensional (2D) models were expanded to threedimensional (3D) models to include the actual slot wall depth. Several parametric variations of the slot geometry, corresponding to various actuator voltages, were investigated. A mass flow rate inlet boundary condition was defined based on the experimentally measured flow rate for a given slot opening. With outlet pressure defined to be atmospheric pressure, a steady state solution was obtained for each valve position. Figure 7 shows the predicted velocity distribution of flow at three different openings. The velocity distribution shows that there is a recirculation zone that forms downstream of the constriction that changes with valve position. The fluidic forces on actuator walls were obtained by integrating pressure values on actuator wall areas. Figure 8 shows the forces on the left wall, right wall and the net force on the actuator. The figure indicates that the forces on the walls of the slots tend to close the actuator, similar to what is observed in the visual observations of the valve oscillation. These forces tend to peak at $14 \mathrm{~V}$.

A Finite Element Model was created to investigate the forcedeflection behavior of the electrothermal beam and understand the


Figure 7. Velocity $(\mathrm{m} / \mathrm{s})$ flow patterns within a single slot at a variety of openings $(X)$ showing a recirculation zone on the left wall.

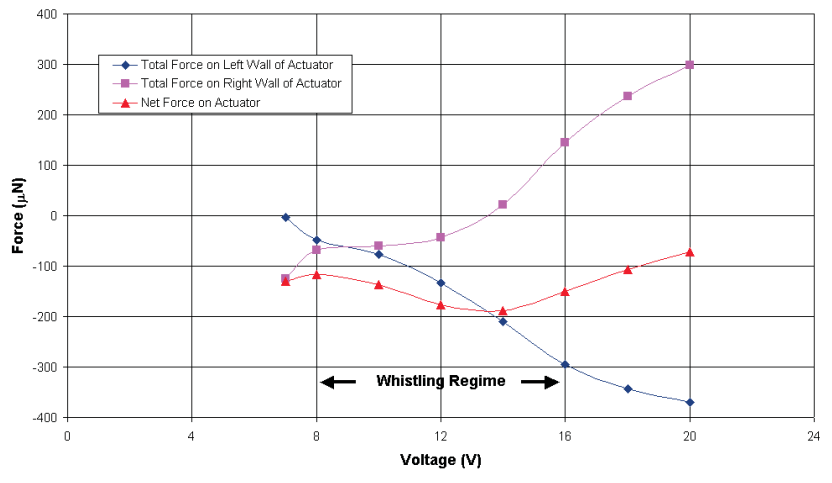

Figure 8. Summary of results from the CFD analysis of a single gas valve slot showing maximum force acting to close the valve at $14 \mathrm{~V}$.

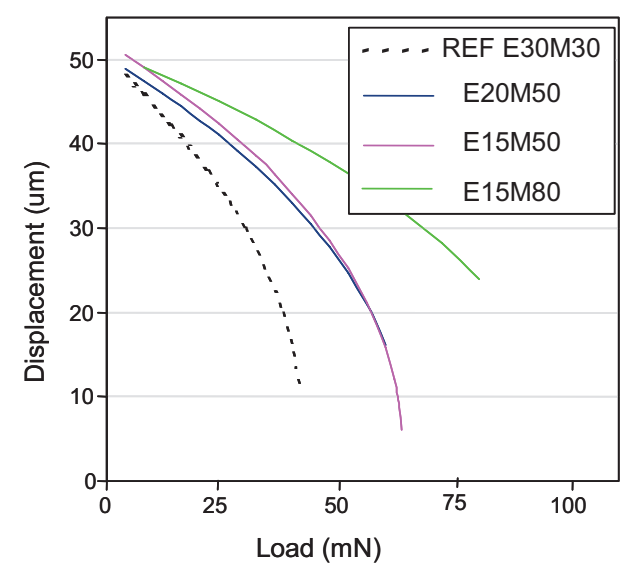

Figure 9. Buckling results for electrothermal beam.

effects of the loads from the fluid flow. The large deflection and snap-through buckling characteristics of the beam required a nonlinear analysis. Since this increased the computational effort, a symmetric, 2D model of the beam was used to reduce simulation time. This facilitated the investigation of many different parametric variations to optimize the characteristics of the electrothermal beam. The beam was simulated by applying an elevated temperature resulting in a net free displacement, similar to the actual electrothermal behavior when electrical current is applied. Next, an opposing force, representing the forces from the fluid flow, is applied that reduces the total displacement of the actuator. The stiffness of the beam is calculated once the displacement and forces are known. The opposing force is then increased until snap-though buckling occurs as the stiffness goes to zero. An example of the force-deflection and buckling characteristics of various electrothermal beams are shown in Figure 9. Here, the legend indicates a parametric investigation of different electrothermal beam widths at the ends "E" and middle "M" in microns. Clearly, the beam can be designed to higher load carrying capability and stiffness while keeping the length constant. A similar 3D model was constructed to investigate variations in the out of plane geometry. For example, variability in fabrication of the beam, especially due to undercutting during the etching process, created beams with an hourglass cross-section. The 3D model showed that the loss of material due to undercutting led to a significant reduction in stiffness of the beam that exacerbated issues related to fluid structure interaction.

\section{SILCONE DAMPING SOLUTION}

Once it was determined that the undesirable excitation, flow instability and noise was due to the FSI, several concepts were explored to increase damping, or reduce the Q-factor of the microvalve. Many of these concepts involved springs attached to slider structure in order to dissipate energy from the motion of the valve. However, a classical solution of an integrated silicon spring attached to the slider would require a very low stiffness in order not to artificially limit the deflection of the actuator and increase the power consumption of the valve. Another possibility not explored here would be to decrease the gap $(5 \mu \mathrm{m})$ between the slots underneath the slider and the slider itself. A smaller gap would increase damping on the device. A similar effect could be accomplished by adding a third wafer with either a corresponding set of slots on top of the actuator [9].

A less risky and more cost effective approach was to attach an elastic, flexible viscoelastic spring to the slider after assembly. Another Finite Element Model was created using ANSYS to investigate the mechanical behavior of the viscoelastic silicone straps used to increase damping of the structure. The geometry of 
the strap used in the model included portions that are bonded to the slider and die edge, and also the region of the strap that is stretched when the slider moves. Static displacement constraints were imposed on the slider, and the opposing force from the strap was determined from the model. This enabled a calculation of an effective spring constant for various sizes and numbers of straps. This spring constant was subsequently introduced into the electrothermal beam actuator model and facilitated sizing of the straps such that the effect on the slider displacement due to the opposing forces of the straps was less than 5\%. The stresses experienced by the strap were found to be small enough to avoid issues related to fatigue such as relaxation or debonding. The model and example stress results are shown in Figure 10. The elastic modulus for the family of silicones considered for the straps is characterized by a storage modulus (real part) and loss modulus (imaginary part). Although the loss modulus is ignored for static deflection, it increases significantly at high frequency such that at the resonant frequency of the valve, it is nearly the same magnitude as the storage modulus. Calculations based on the strap model showed that the viscous forces due to the strap could easily exceed the elastic forces. This finding gave a good indication that the viscoelastic properties of the silicone provided the damping force needed to eliminate the undesirable FSI.

A GE RTV106 paste was used and dispensed on the microvalve on two opposite locations as seen in Figure 11. RTV106 was chosen due to high temperature capability and its viscosity to prevent flow down into the slots of the microvalve. The RTV106 was dispensed using a MRSI 170G auto dispense system after the valves were attached to the boards. This tool used vision recognition to accurately apply the two straps. The final straps were $300 \mu \mathrm{m}$ wide and extended approximately 800 $1000 \mu \mathrm{m}$ in length. Valves with silicone straps were tested as part of the flow manifold to determine the effect of these straps on the valve's reliability. The valves were actuated from a closed to open position at a frequency of $1 \mathrm{hz}$. Tests of 5 devices indicated no failure when cycling up to 10,000 cycles.
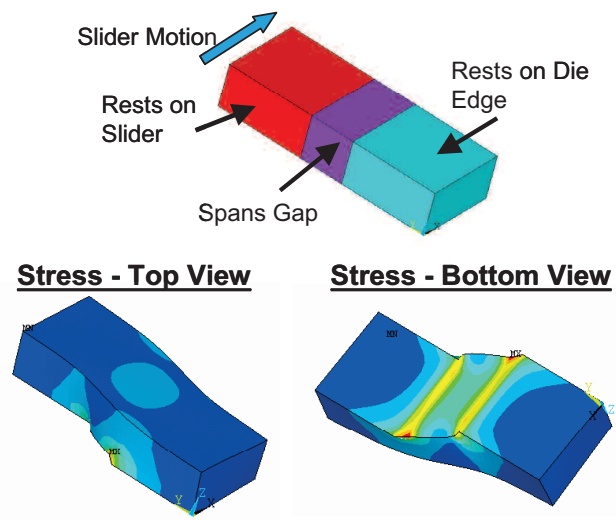

Figure 10. Stress and design analysis of silicone strap used for damping.

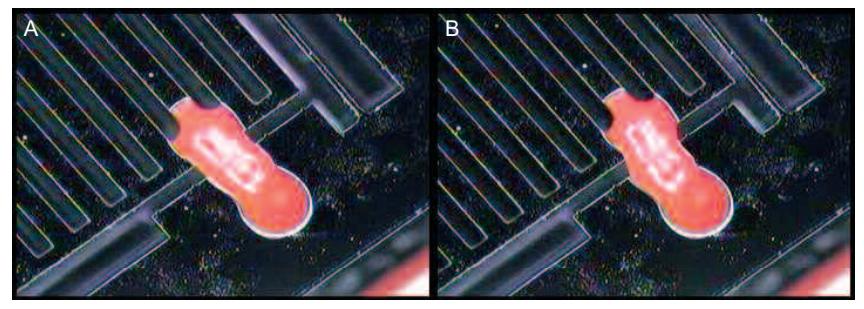

Figure 11. Optical photographs of silcone "strap" used to dampen the flow induced oscillation of the gas valve. A, shows the valve at $0 \mathrm{~V}$ and $B$ shows the valve $24 \mathrm{~V}$.

\section{CONCLUSIONS}

A high flow rate gas valve was designed, fabricated, and tested. Initial testing of the silicon valve indicated a flow-induced resonance which caused unstable motion of the electrothermal actuators in the plane of the wafer. This resonance of the electrothermal beams caused undesirable and inconsistent flow/voltage response of the valve. In order to understand the origin of the resonance, a CFD model of an individual slot at various positions indicated that flow forces act to close the valve. An analysis of the forces necessary to buckle the thermal beams revealed that the flow forces are insufficient on their own to cause the deflections seen experimentally. However, due to the low damping of the slider and electrothermal beams, these small flow induced oscillations are amplified into large deflections. In order to prevent the resonance of the structure, damping was added through the addition of a pair of silicone springs to the slider. Testing of these devices indicated that the resonance of the structure was removed with the addition of the silicone dampers. Limited testing of the reliability indicated that these dampers were reliable up to 10,000 cycles.

\section{ACKNOWLEDGMENTS}

The authors would like to acknowledge the support and hard work of the team at GE-Infrastructure Sensing including George $\mathrm{Wu}$, Sisira Gamage, Terry Cookson and Sam Wong who fabricated the microvalve devices. The authors would like to thank Jason Castle for microvalve testing and Don Lester for work in die attach and RTV dispense. The authors also wish to acknowledge the support of Todd Graves, Dave Najewicz, and Nick Okruch.

\section{REFERENCES}

[1] R.E. Mihailovich, et. al., "Dissipation measurements in vacuum-operated single-crystal silicon microresonators", Sensors \& Actuators A, vol. 50, pp. 199-207, (1995).

[2] K.Y. Yasumura, et al., "Quality factors in micron- and submicron-thick cantilevers", Journal of MEMS, vol. 9, no. 1, pp. 117-125, (2000).

[3] Y.H. Cho, et al., "Viscous Energy Dissipation in Laterally Oscillating Planar Microstructures: A Theoretical and Experimental Study", Proc. of IEEE MEMS, Ft. Lauderdale, FL, (1993).

[4] F.R. Blom, et al., "Dependence of the quality factor of micromachined silicon beam resonators on pressure and geometry", J. Vac. Sci. Technol., B10, 1, pp. 19-26, (1992).

[5] K. Y. Yasumura, J. D. Grade, and H. Jerman, "Fluid Damping of an Electrostatic Actuator for Optical Switching Applications," Proc. of the 2002 Solid State Sensor and Actuator Workshop, Hilton Head, SC, pp. 358-61, (2002).

[6] M. Walters, et. al., "A silicon micromachined gate valve," presented at the 1998 Solid State Sensors and Actuators Workshop, Hilton Head, SC, (1998).

[7] A. Knobloch, et. al., "Experimental Study of Entrance Effects on Laminar Gas Flow through Silicon Orifices", Proc. of 2005 ASME International Mechanical Engineering Congress and Exposition (IMECE), Orlando, November 5-11, (2005).

[8] K. R. Williams, et. al., "A Silicon Microvalve for the Proportional Control of Fluids," Digest of the 10th International Conference on Solid-State Sensors and Actuators (Transducers '99), Sendai, Japan, pp. 1804-1807, (1999).

[9] K. Y. Yasumura and H. Jerman, "Pressure Enhanced Air Damping in Enclosed Laterally Oscillating Microstructures," submitted to the 2006 Solid State Sensor and Actuator Workshop, Hilton Head, SC, (2006). 